\title{
BMJ Open Quality Improving the waiting times within a hospice breathlessness service
}

\author{
Caroline Sime, ${ }^{1}$ Stuart Milligan, ${ }^{2}$ Kevin Donal Rooney ${ }^{2,3}$
}

\begin{abstract}
To cite: Sime C, Milligan S, Rooney KD. Improving the waiting times within a hospice breathlessness service. BMJ Open Quality 2019;8:e000582. doi:10.1136/ bmjoq-2018-000582
\end{abstract}

Received 19 November 2018 Accepted 20 November 2018

Check for updates

C Author(s) (or their employer(s)) 2019. Re-use permitted under CC BY-NC. No commercial re-use. See rights and permissions. Published by BMJ.

${ }^{1}$ Institute for Research in Healthcare Policy \& Practice, University of the West of Scotland, Hamilton, Scotland ${ }^{2}$ School of Health and Life Sciences, University of the West of Scotland, Paisley, Scotland ${ }^{3}$ Consultant in Anaesthesia and Intensive Care Medicine, Royal Alexandra Hospital, Paisley, Professor of Care Improvement, University of the West of Scotland, Hamilton, Scotland

Correspondence to Dr Caroline Sime; caroline.sime@uws.ac.uk

\section{ABSTRACT}

Background Breathlessness, a common symptom in advanced disease, is a distressing, complex symptom that can profoundly affect the quality of one's life. Evidence suggests that specialist palliative care breathlessness intervention services can improve physical well-being, personal coping strategies and quality of life. In the UK, the use of quality improvement methods is well documented in the National Health Service. However, within the independent hospice sector there is a lack of published evidence of using such methods to improve service provision.

Aim The aim of this project was to reduce the waiting time from referral to service commencement for a hospice breathlessness service by $40 \%$-from a median of 19.5 to 11.5 working days.

Methods Using a quality planning and systems thinking approach staff identified barriers and blockages in the current system and undertook plan-do-study-act cycles to test change ideas. The ideas tested included offering home visits to patients on long-term oxygen, using weekly team 'huddles', streamlining the internal referral process and reallocating staff resources.

Results Using quality improvement methods enabled staff to proactively engage in positive changes to improve the service provided to people living with chronic breathlessness. Offering alternatives to morning appointments; using staff time more efficiently and introducing accurate data collection enabled staff to monitor waiting times in real time. The reduction achieved in the median waiting time from referral to service commencement exceeded the project aim.

Conclusions This project demonstrates that quality improvement methodologies can be successfully used in a hospice setting to improve waiting times and meet the specific needs of people receiving specialist palliative care.

\section{INTRODUCTION}

\section{Background}

Breathlessness is a multidimensional and subjective symptom. It is often described by the physical, psychological and social impact it has on one's daily life, such as causing exhaustion, increased anxiety and social isolation. ${ }^{1-4}$ Its onset is usually insidious and can be a symptom of serious, life-limiting illness including cancer, heart failure and non-malignant lung disease. ${ }^{56}$

The successful use of a holistic approach used by specialist palliative care services to meet the physical, psychological and social dimensions of any life-limiting illness is well evidenced. $^{7-9}$ Furthermore, specialist palliative care services focusing on the management of breathlessness have been found to meet the needs of those experiencing life-limiting breathlessness. ${ }^{10-12}$ In particular, there is good evidence for interventions to manage breathlessness such as breathing exercises, relaxation techniques, handheld fans and optimisation of pharmacological interventions and psychological and social support. ${ }^{13}$ However, the provision of these services across the UK remains inconsistent and the services are often underused by those most in need. This can be attributed to a lack of integration of these specialist services with existing, disease-specific services ${ }^{12}$ and negative perceptions held by people with end-stage non-malignant disease who perceive specialist palliative care, or hospice care, as for people dying of cancer. ${ }^{11}$

Across the UK, within the National Health Service (NHS), it has been recognised that to meet the growing demands of the population there needs to be a commitment to continuous quality improvement $(\mathrm{QI})$ as its fundamental strategy. ${ }^{145}$ Yet, despite hospice services facing the same growing demands on their services, there is little published evidence of QI initiatives being undertaken within the independent hospice sector. The goals of this project were to successfully use QI methodology within a hospice environment to improve the timeliness of the care and the experience of people accessing a breathlessness intervention service.

\section{Setting and context}

Situated in the West of Scotland, Ardgowan Hospice is an independent, third sector organisation that provides palliative care services within the local Health and Social Care Partnership (HSCP) of Inverclyde, which serves a population of approximately 80000 . The HSCP is under the strategic leadership and guidance of NHS Greater Glasgow \& Clyde, Scotland's largest NHS territorial health board. In December 2015, the Scottish Government published its vision for 
developing palliative care services stating that anyone in Scotland who requires palliative care, should have access to it 'regardless of age, gender, diagnosis, social group or location' (p. 3 Scottish Government, 2015). ${ }^{16}$

Ardgowan Hospice has provided specialist palliative care services for over 30 years and receives approximately 500 new referrals each year. It is a charitable organisation (similar to most hospices in Scotland) and receives approximately $40 \%$ of its funding from the local HSCP. It relies on donations and fundraising to meet the remaining costs of providing services. The hospice is governed by The Clinical Standards Board for Scotland standards for specialist palliative care services. ${ }^{17}$ These standards have not been substantially updated for 15 years but are subject to review as part of the Strategic Framework for Action for Palliative Care in Scotland. ${ }^{16}$ In the absence of regularly updated standards, practice developments need to be subject to strict local governance. ${ }^{18}$ At Ardgowan Hospice, the past 15 years have been characterised by numerous new practice and service initiatives. While this level of innovation is to be commended, nevertheless a structured and standardised approach is needed for implementation of new practice and the model for improvement provides the opportunity to deliver service improvements using an evidence-based and systematic approach. ${ }^{19}$

\section{Local problem}

Modelled on the Cambridge Breathlessness Intervention Service,$^{20}$ the hospice breathlessness service offers a multidisciplinary approach to the management of chronic breathlessness for people living with any life-limiting illness using a variety of interventions including non-pharmacological interventions, patient and family education and support and bespoke exercise programmes. ${ }^{21}$

The service is managed by the hospice physiotherapist and is supported by specialist trained nurses. Together, they offer an assessment and plan of care for both the physical and psychosocial needs of the patient and their carer. In recent months however, due to staff turnover and high absence rates, staff availability to provide this service had been limited. In addition, there had been an increase in referrals of people with more advanced disease and complex needs.

In contrast to other hospice services, the breathlessness service median waiting time from referral to service commencement was found to be six working days in 2014, increasing, and sustained, to 19.5 working days between January and August 2015. Analysis of this service found no standardised internal referral process, incomplete data collection and inconsistent staffing arrangements.

\section{METHODS}

\section{Improvement team}

In January 2015, in collaboration with the University of the West of Scotland, the hospice launched a new initiative to promote a culture of continuous improvement within all hospice services and systems, using the model for improvement as its methodology.

A Data Analyst was recruited to the team to improve data collection and usage within the hospice, and a Research Fellow was appointed to develop a culture and practice of continuous QI. The overall aim of the programme was twofold: to investigate and improve the hospice patient's journey, particularly with regard to optimising timing of referral and referral-to-admission time, and to develop a culture of improvement within the hospice. Support and guidance for the programme had been agreed via a memorandum of understanding between the two organisations, and was led by an experienced improvement lead and Scottish Patient Safety Fellow from the university.

\section{Intended improvement}

Using a quality planning and systems thinking approach current referral pathways, processes and clinical practice were explored with the administrative and clinical teams who managed referrals and worked within the breathlessness service. The patient pathway was segmented into the referral process and the clinical management of the service.

First, using a process map to identify blockages and barriers to the referral process, the team identified areas of potential improvement. ${ }^{19}$ There was a lack of operational definitions to facilitate consistency in key measurements including the date of referral start time (eg, date sent from referrer and date received by the hospice were used interchangeably); and there was no administrative support to organise the booking of the breathlessness clinic appointment or transport if required.

Second, using a cause and effect model (figure 1), the clinical team identified among other things, a lack of flexibility in appointment times to meet the needs of patients with complex needs, including those on long-term oxygen therapy and people living with high levels of anxiety and distress secondary to their chronic breathlessness as well as issues around the staffing of the service.

Through regular meetings with the administrative clinical support team and clinical teams, operational definitions were standardised to ensure referral dates recorded were consistent (date of referral was defined as the date the referral was received and processed by the hospice clinical support team). A driver diagram (figure 2), and a project charter were created; and an ambitious, but realistic, aim statement was developed.

By June 2016, there will be a $40 \%$ reduction in the time people are waiting from referral to service commencement of the breathlessness service (a reduction in the median from 19.5 to 11.5 working days).

The outcome measure chosen was the length of time from referral date (date the referral was received and processed by the clinical support team) to service start date (the date of the first assessment). Furthermore, agreement was reached to count working days only. By doing so, this reflected the current national clinical standards for specialist palliative care services. ${ }^{17}$ The process 


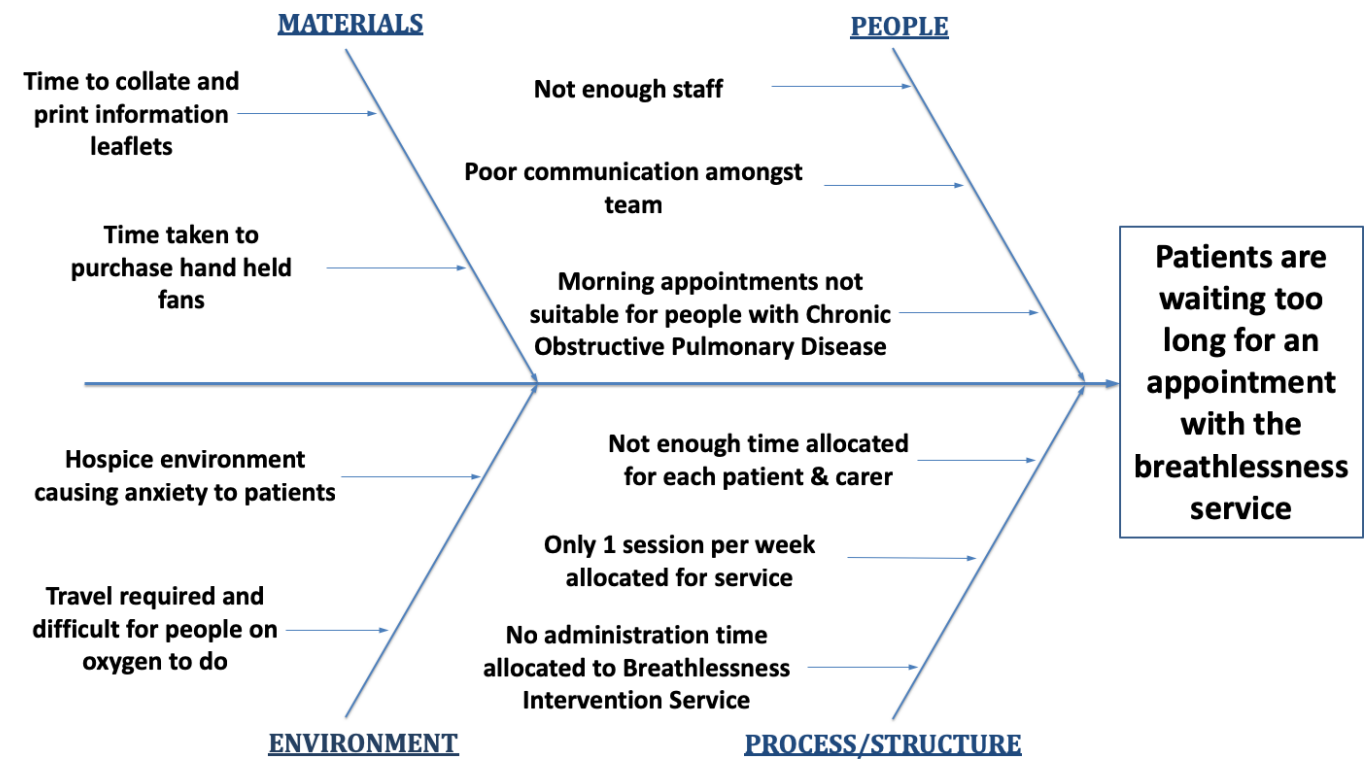

Figure 1 Cause and effect diagram created with the multidisciplinary team of the reasons for why there are delays in the provision of the breathlessness service to patients.

measures were ensuring there was compliance with having two members of staff available to work within the service each week, and the number of fully completed patient assessments per week. These measures were chosen because without a full complement of staff, at times, assessments were only partially completed. The physiotherapist who managed this service, was responsible for documenting completed assessments, and the QI researcher was responsible for retrieving the data on waiting times from the clinical support team, and documenting staff numbers weekly.

Constraints on this project included no additional funding for additional clinical staff hours or increased administrative support. To ensure a more efficient use of hospice staff resource, the current workforce capacity was analysed. The staff identified with capacity to undertake at least one 2 hour assessment per week and who had the skills and experience to assist the service were

\section{Project Aim}

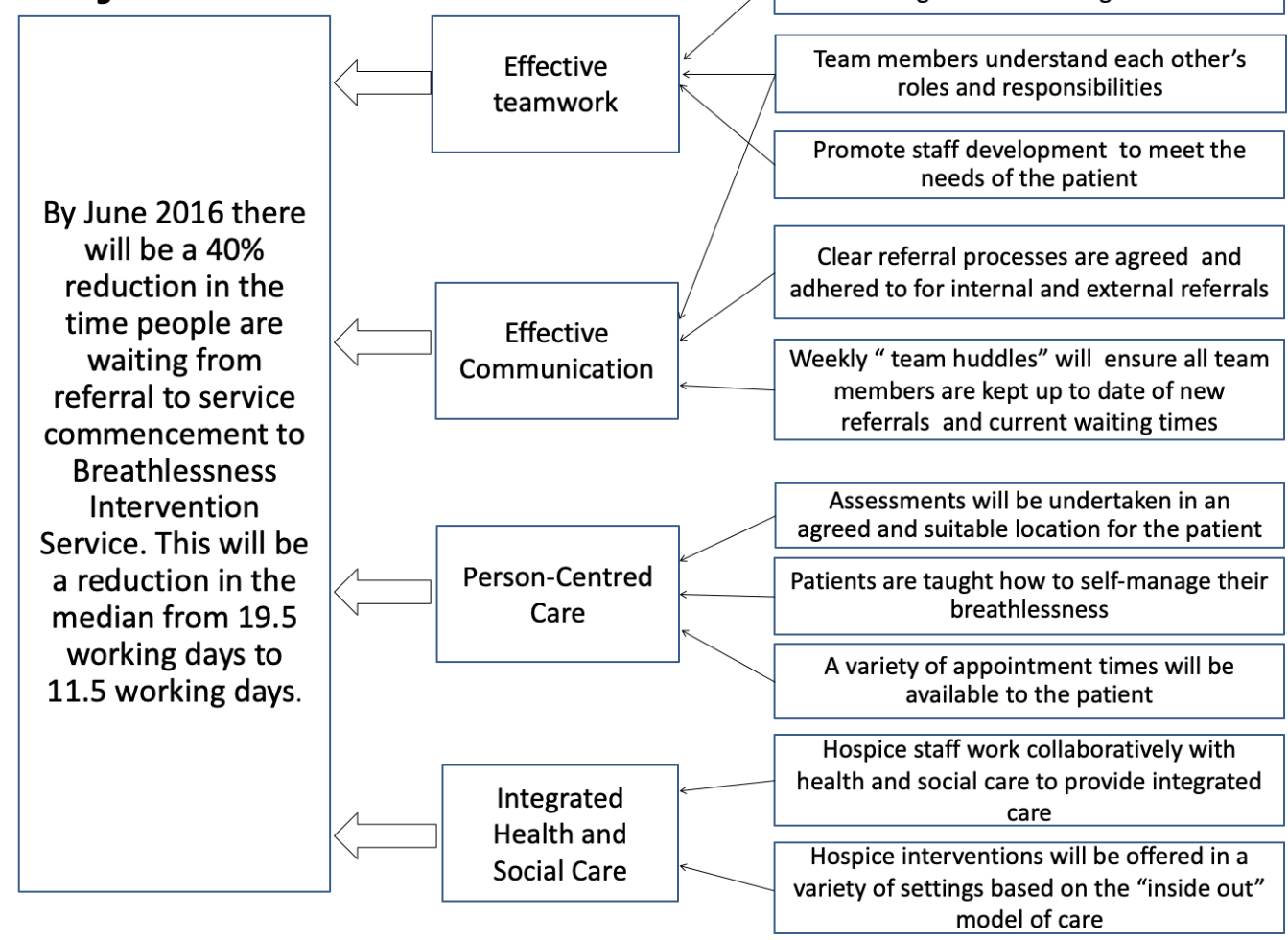

Figure 2 Overarching project driver diagram highlighting the key drivers and change ideas identified and agreed by the team. 
the occupational therapist and family support worker (an experienced specialist nurse). A balancing or unintended consequence measure was to measure time spent by these individuals away from the inpatient unit in order to ensure that the assistance of these staff was not to the detriment of their core work and other patient care.

The lack of robust and recently updated clinical standards for specialist palliative care services made it difficult to engage staff in the improvement of a service that had already met the national standard. For example, the national standard is that services should make initial contact within one working day of receiving a referral. There is no standard to state when services start, therefore a person living with chronic breathlessness could be advised their referral had been received yet wait an indefinite period to get an appointment. To overcome this hesitance by staff, a climate and urgency for change ${ }^{22}$ were created using patient stories and visualisation of waiting times using run charts.

\section{Ethical consideration}

This QI project met the exemption criteria of the hospice and NHS for ethical review because the work was considered service improvement and not human subject research.

\section{INTERVENTIONS}

The primary outcome measure was the length of time from referral date to service start date for the breathlessness intervention service. Change ideas to be tested in the plan-do-study-act (PDSA cycles were:

1. Reassign staff from the wider clinical team to support the breathlessness intervention service (BIS).

2. Offer home visits to patients with high levels of anxiety and those on long-term oxygen therapy as this had been shown to prevent them from attending the hospice.

3. Introduce weekly 'huddles' to ensure all staff members were aware of patients waiting and progress of those attending the service.

4. Introduce guidelines for internal referrals that were currently unaccounted for within the data collected.

\section{Recruiting staff}

A key priority that emerged from the investigative work was to address the lack of staff that supported BIS. Two key members of staff provided this service, and whenever a staff member was not available, the service simply did not run. The natural development of services at the hospice had created silos of work, within which staff had not recognised the need to break down or work across services to provide holistic, person-centred care.

By analysing the workforce capacity and identifying the skills and attributes which staff were required to have, the team were able to identify staff in the wider multidisciplinary team who also had these skills and could potentially assist the delivery of the service during periods of absence of the core team. Therefore, qualified nurses from out with day services (eg, the family support worker and the specialist community nurses) were identified to cover for the day service nurse, and the occupational therapist was identified to cover for the physiotherapist. Staff were approached and invited to assist at this clinic. This created a larger core team of staff who provided the BIS, and weekly monitoring of staff numbers identified an improvement in regular cover for the service.

Four PDSAs were undertaken to improve the staff availability for BIS. Each test focused on the availability of staff at the time referrals were received and how easily they could facilitate appointments. The findings from this change idea then contributed to the change idea to offer flexible appointments and home visits to improve meeting the needs of the patient and service demands elsewhere in the system.

\section{Home visits versus clinic}

Exploration of the waiting time data had identified a changing demographic of people referred for BIS. When the service was originally set up, all of the referrals were for people with lung cancer. Now, $50 \%$ of referrals were for people living with non-malignant lung disease. The needs of people with non-malignant disease are very similar to those with lung cancer ${ }^{6}$; however, staff experiences of working with people with non-malignant lung disease indicated their breathlessness was worse in the morning, they often took longer to get washed and dressed and were often too weak and exhausted after getting dressed to attend morning appointments. In addition, for those on long-term oxygen use, identifying appropriate transport and organising a time to attend appointments often led to long delays.

It was agreed that in order to reduce the waiting time for these patients, home visits would be an appropriate change idea. It would reduce staff time spent organising transport and reduce the number of wasted appointments due to failure to attend morning appointments.

Three PDSAs were undertaken to test this change idea and all three found home visits were positively received by patients and their carers. While BIS always evaluated strongly from patients feedback, offering home visits appeared to subjectively reduce fear and anxiety regarding their referral to a hospice.

\section{Weekly 'huddles'}

At the start of this project, staff were unaware that there was a problem with their waiting times. Without receiving real time feedback, they did not know how many people they had waiting to be seen and what their current service waiting times were. This information was held centrally and as long as initial contact was made with the patient within one working day (to meet the national clinical standards), there was no further prompting from the clinical support team therefore, long waiting times could remain undetected.

Staff agreed a weekly 'huddle' would be appropriate to review this information and also serve to share clinical 
updates of people currently seen by BIS. The huddle, or debrief, derived from patient safety initiatives, enables staff to meet at a key point in the day (or in this case, a week) to discuss and prioritise issues and manage risk. The aim of these huddles was to spend fifteen minutes reviewing the data and discussing current patients. Six PDSAs were undertaken to identify the most appropriate time and day to undertake the huddle, and to refine the process to ensure the huddle lasted no longer than the $15 \mathrm{~min}$ as initial testing found staff would often use the opportunity to discuss wider issues and would lose focus.

\section{Internal referral process}

As this project progressed, there was a downward, and improving trend in waiting times. Staff engagement and enthusiasm for the project was good and then without explanation, waiting times started to increase. The data were checked and case note review found no obvious reason to explain this rise in waiting times. However, exploring this at the weekly huddle it was found that staff often sent internal referrals to the service. These were either done during an informal discussion, phone call or email, and therefore the patient was not documented as waiting for the breathlessness service. Their omission from any data capture for this service did not routinely impact on waiting times until the number of referrals increased and therefore lengthened the waiting times of referrals who had been recorded.

Working with the clinical support team, a simple, tick box form was developed, tested and a process agreed among all clinical staff. The form was not to detract from face-to-face discussions regarding referrals, but to capture the referral information in a timely and efficient way. This form and process underwent twelve PDSA cycles during the project. Tests of change focused on developing the information captured on the form, the processing of the form via the daily referrals meeting to the administrative staff and the integration of the form with current patient records.

\section{RESULTS}

The study period for this project was August 2015May 2016. The number of referrals to the BIS was 23, including internal hospice referrals. The aim was to reduce the waiting time from referral to commencement of the breathlessness intervention service by $40 \%$, from a median of 19.5 to 11.5 working days. The improvement achieved exceeded this, and a sustained improved median waiting time of 9.5 days was achieved. A run chart was used due to the initial small patient numbers and to record the data in a way that staff would easily understand (figure 3).

Throughout the project, through staff reallocation, the number of staff available to assist the service improved and over time the staffing of the service became stable with additional support to cover any absence from the core team. The second process measure, counting the number of completed holistic assessments revealed an improvement in the time taken to complete the two-part assessment as staff coordinated their assessments and developed care plans, usually within 2 days for the two parts. Furthermore, there was no impact detected on staff time away from the inpatient unit as there was less time spent carrying out administrative duties, such as arranging complex transport schedules. Staff also reported greater satisfaction undertaking assessments in patients' own homes, as they were able to capture more detailed information to improve the quality of their assessment.

\section{DISCUSSION}

There is little published evidence available outlining the use of QI methods in a UK hospice setting. It has been suggested that hospice teams face unique challenges to using QI because they offer a small range of clinical services, the lack of QI training within the hospice sector and the multidisciplinary design of the clinical team. ${ }^{23}$ Comparisons can be drawn with carrying out research involving people receiving palliative care. There is

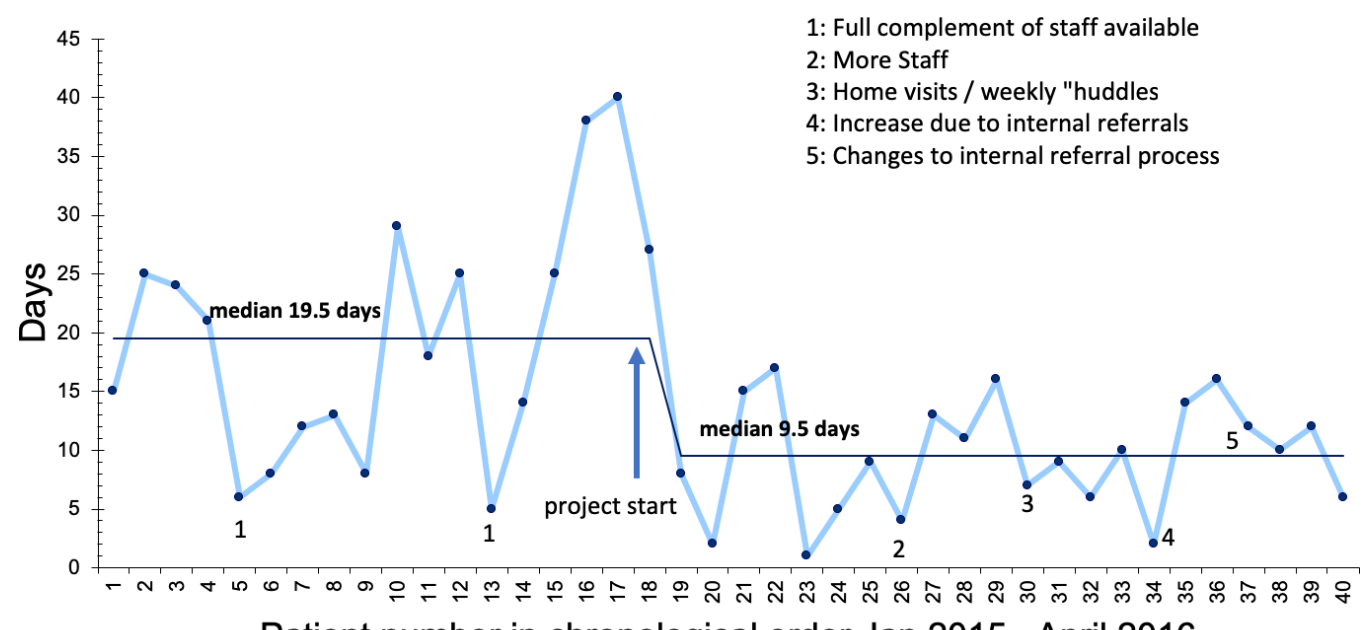

Patient number in chronological order Jan 2015 - April 2016

Figure 3 Run chart demonstrating the patient waiting times (including baseline data) from the project commencing in August 2015-April 2016 from referral to service start time for the breathlessness service. 
evidence to suggest that there are practical and ethical difficulties in undertaking research with this group, and often staff prevent access to these patients who are often seen as the most vulnerable research subjects. ${ }^{24-26}$ This is despite the fact that palliative care patients often welcome the opportunity to be involved in research and report that they want to make a contribution. ${ }^{27}$ A lack of understanding of QI may prevent palliative care staff using this methodology for fear of making things worse for patients and causing more distress. Furthermore, all the opposition to using palliative care recipients as research subjects must be weighed against the argument that it is actually unethical not to involve this patient group. ${ }^{24}$ It can be argued that it is also unethical to omit specialist palliative care services from service improvement activities that will benefit staff and most importantly, patients and their families.

This project demonstrates the successful utilisation of QI methods that led to an improvement in the timeliness and care offered to people living with breathlessness in a hospice care setting. Similar to findings by Ross and Naylor ${ }^{28}$ who highlighted the effective use of QI in mental health services, this project supports their assertion that whatever the clinical area requiring improvement, if there is strong leadership, organisational readiness and honest and transparent reporting, QI methods can be used in any setting. This relies on the workforce sharing the belief that change is not only required but is achievable, and has the resources to deliver change. ${ }^{22} 2930$

In this project, the ambition of the hospice was to ensure that anyone with unmet needs could access the service where, when and how they needed it. Therefore, the change ideas of offering assessments faster and giving the patient a choice of where the assessment was to be undertaken, reflected the core values of the hospice and facilitated staff to embrace these changes. In addition, there was also organisational support at all levels for the project, and the clinical team played key roles in setting the project objectives, identifying areas for improvement and testing the change ideas. Furthermore, having successful outcomes can in part be attributed to the lead practitioner working as an autonomous practitioner. This enabled the project to be led and developed within a system that supported space for creativity and growth that emerged from an urgency to improve the patient experience. While this small improvement project cannot be judged as a social movement, it does reflect the key attributes (such as creating an urgency by focusing on the patient experience, driven by a desire for change and led by the clinical team) of a successful social movement. ${ }^{31}$ An improved waiting time and patient experience in this service have contributed to the continuous improvement model being adopted by the wider hospice team, and is evidenced in the ongoing improvement journey that has developed across a number of services since the completion of this project. This has included a new service model to reach more people living with life limiting conditions in their own communities and homes, and working towards meeting the national objectives set out in the strategic vision for palliative care services. ${ }^{16}$

\section{Strengths and limitations}

The main strength of this project can, from another perspective also be considered a limitation. This project was undertaken in a small regional hospice, thus enabling the project team to appreciate the wider system that the breathlessness service operated within. We engaged directly with the clinical and administrative teams directly working with the service and communicated and advised the wider teams of the hospice of the project and its progress.

In turn however, the scale of this project was small, having 23 referrals made to this service over the period of the project. Furthermore, it is not known how much of this project could be replicated in another hospice because as independent, charitable organisations, each hospice is managed in a unique way to meet the needs of its own community. In this project, setting operational definitions to standardise the collection of data contributed to an improvement in the recorded waiting times. This improvement was the result of a reduction in variation rather than any test of change. However, this reflects the weaknesses in the current standards hospices adhere to, including the use of working days for measurement, and there needs to be a wider debate and agreement on setting national operational definitions to improve the quality of data currently collected by hospices across Scotland. Furthermore, the improvement described here was partly achieved through the re-allocation of hospice resources to the breathlessness service. A question mark remains over the long-term sustainability of the improvement if those resources become unavailable at some point in the future. Therefore, every effort must be made to embed the new way of working in routine practice (through the creation of standard operating procedures and/or policies).

\section{CONCLUSION}

This project has demonstrated that it is possible to introduce and facilitate a culture of improvement within the independent hospice environment. It has shown that the various methodologies used are applicable to the hospice setting and that measurable improvements can be achieved with a structured and systematic approach. The staff involved in the service adapted readily to the QI approach due to the support of the senior management team, which was essential to the success of the project.

The project delivered specific benefits for patients in terms of reduced waiting times. There were also other potential benefits which were not assessed including greater flexibility of service and improved patient experience.

This paper contributes to the limited evidence of QI initiatives in a UK hospice setting. Without such evidence, there may be a reticence to embrace QI within this sector 
as a valid and robust method to improve services and ultimately the patient experience.

Acknowledgements This project would not have been successful without the full support of the senior management team at Ardgowan, the clinical and administrative teams and the patients accessing hospice services, often for the first time.

Contributors Conception or design of the work was undertaken by all authors (CS, $\mathrm{KDR}, \mathrm{SM})$. The quality improvement project was undertaken by CS, and supervised. by SM and KR. Drafting the article was undertaken by CS and critical revision of the paper by KR and SM. Final approval of the version to be published was given by KR.

Funding This quality improvement project was jointly funded by Ardgowan Hospice and the University of the West of Scotland.

Competing interests None declared.

Patient consent for publication Not required.

Provenance and peer review Not commissioned; internally peer reviewed.

Open access This is an open access article distributed in accordance with the Creative Commons Attribution Non Commercial (CC BY-NC 4.0) license, which permits others to distribute, remix, adapt, build upon this work non-commercially, and license their derivative works on different terms, provided the original work is properly cited, appropriate credit is given, any changes made indicated, and the use is non-commercial. See: http://creativecommons.org/licenses/by-nc/4.0/.

\section{REFERENCES}

1. Society AT. Dyspnoea: Mechanisms, assessment and management. A consensus statement. American Journal of Respiratory and Critical Care Medicine 1999;159:321-40.

2. Ahmadi NS, Månsson J, Lindblad U, et al. Breathlessness in everyday life from a patient perspective: a qualitative study using diaries. Palliat Support Care 2014;12:189-94.

3. Gysels M, Higginson IJ. Access to services for patients with chronic obstructive pulmonary disease: the invisibility of breathlessness. $J$ Pain Symptom Manage 2008;36:451-60.

4. Dunger C, Higginson IJ, Gysels M, et al. Breathlessness and crises in the context of advanced illness: A comparison between COPD and lung cancer patients. Palliat Support Care 2015;13:229-37.

5. Weingaertner V, Scheve C, Gerdes V, et al. Breathlessness, functional status, distress, and palliative care needs over time in patients with advanced chronic obstructive pulmonary disease or lung cancer: a cohort study. J Pain Symptom Manage 2014:48:569-81.

6. Currow DC, Smith J, Davidson PM, et al. Do the trajectories of dyspnea differ in prevalence and intensity by diagnosis at the end of life? A consecutive cohort study. J Pain Symptom Manage 2010;39:680-90.

7. Higginson IJ, Evans CJ. What is the evidence that palliative care teams improve outcomes for cancer patients and their families? Cancer J 2010;16:423-35.

8. Sampson EL, Ritchie CW, Lai R, et al. A systematic review of the scientific evidence for the efficacy of a palliative care approach in advanced dementia. Int Psychogeriatr 2005;17:31-40.

9. Gadoud AC, Johnson MJ. Palliative care in non-malignant disease. Medicine 2011;39:664-7.

10. Farquhar MC, Prevost AT, McCrone P, et al. The clinical and cost effectiveness of a Breathlessness Intervention Service for patients with advanced non-malignant disease and their informal carers: mixed findings of a mixed method randomised controlled trial. Trials 2016;17:185.

11. Hayle C, Coventry PA, Gomm S, et al. Understanding the experience of patients with chronic obstructive pulmonary disease who access specialist palliative care: a qualitative study. Palliat Med 2013;27:861-8.

12. Higginson IJ, Bausewein C, Reilly CC, et al. An integrated palliative and respiratory care service for patients with advanced disease and refractory breathlessness: a randomised controlled trial. Lancet Respir Med 2014;2:979-87.

13. Chin C, Booth S. Managing breathlessness: a palliative care approach. Postgrad Med J 2016;92:393-400.

14. Ham C, Berwick D, Dixon J. Improving Quality in the English NHS. The King's Fund. 2016 https://www.kingsfund.org.uk/sites/default/ files/field/field_publication file/Improving-quality-Kings-FundFebruary-2016.pdf (Accessed 30 Nov 17).

15. Dayan M, Edwards N. Learning from Scotland's NHS. Research report. Nuffield Trust. 2017.

16. Scottish Government. Strategic Framework for Action on Palliative and End of Life Care. 2015 http://www.gov.scot/Resource/0049/ 00491388.pdf (Accessed 27 Nov 17).

17. Clinical Standards Board for Scotland. Clinical Standards Specialist Palliative Care. 2002. Available at https://www.palliativecarescotland. org.uk/content/publications/ClinicalStandardforSPC.pdf (Accessed 27 Nov 2017).

18. McSherry R. Practice development and health care governance: a recipe for modernization. J Nurs Manag 2004;12:137-46.

19. Langley GL, Moen R, Nolan KM, et al. The Improvement Guide: $A$ Practical Approach to Enhancing Organizational Performance. 2nd edition. San Francisco: Jossey-Bass Publishers, 2009.

20. Breathlessness Intervention Service. http://www.cuh.org.uk/ breathlessness-intervention-service-bis/about/what-we-do (Accessed 27 Nov 2017).

21. Booth $S$, Farquhar $M$, Gysels $M$, et al. The impact of a breathlessness intervention service (BIS) on the lives of patients with intractable dyspnea: a qualitative phase 1 study. Palliat Support Care 2006;4:287-93.

22. Kotter JP. Leading Change. $2^{\text {nd }}$ edition. Boston, MA: Harvard Business Review Press, 2012.

23. Kamal AH, Nicolla JM, Power S. Quality Improvement Pearls for the Palliative Care and Hospice Professional. J Pain Symptom Manage 2017:54:758-65.

24. Kaasa S, Forbes K. et a/Research in palliative care. In: Cherny NI, Fallon MT, Kaasa S, Portenoy RK, Currow DC, . eds. Oxford textbook of palliative medicine. 5 th edition. Oxford: Oxford University Press, 2015.

25. Walker S, Read S. Accessing vulnerable research populations: an experience with gatekeepers of ethical approval. Int J Palliat Nurs 2011:17:14-18

26. Kars MC, van Thiel GJ, van der Graaf $R$, et al. de Graeff, A. and van Delden, J.J. (2016) A systematic review of reasons for gatekeeping in palliative care research. Palliative Medicine 2016;30:533-48.

27. Alexander SJ. 'As long as it helps somebody': why vulnerable people participate in research. Int J Palliat Nurs 2010:16:173-8.

28. Ross S, Naylor C. Quality improvement in mental health. The King's Fund. 2017 https://www.kingsfund.org.uk/sites/default/files/field/ field_publication_file/Quality_improvement_mental_health_Kings Fund July_2017_0.pdf (Accessed 30 Nov 2017).

29. Weiner BJ. A theory of organizational readiness for change. Implement Sci 2009;4:67.

30. Willis $\mathrm{CD}$, Saul J, Bevan $\mathrm{H}$, et al. Sustaining organizational culture change in health systems. J Health Organ Manag 2016;30:2-30.

31. Bate P, Robert G, Bevan $\mathrm{H}$. The next phase of healthcare improvement: what can we learn from social movements? Qual Saf Health Care 2004;13:62-6. 